\title{
A Framework for Locomotional Design: Toward a Generative Design Theory
}

\author{
Susanne Jul \\ Electrical Engineering and Computer Science \\ University of Michigan \\ sjul@acm.org
}

\begin{abstract}
Generative design theories are needed to bridge the gaps between pure scientific knowledge, individual ("point") designs and systematic generation of viable design alternatives. This paper suggests a framework for locomotional design that uses knowledge of navigation and spatial cognition to inform design. Examples of the implications of two such pieces of knowledge are sketched out, suggesting how this framework might lead to a generative design theory.
\end{abstract}

\section{KEYWORD}

Theory-driven Design, Informed Design, Design Generation, Generative Design Theory, Design Framework, Navigation, Wayfinding, Locomotion.

\section{INTRODUCTION}

Usability engineering has become a major industry over the past two decades. Methodologies for structuring the design process help d esigners collect and o rganize information relevant to generating good designs, and evaluation techniques help $\mathrm{d}$ esigners choose a mong design alternatives. However, there has been relatively little focus on supporting generation of good design alternatives by informing design content systematically. Thus, designers often expend significant effort in "rediscovering the wheel" or, worse yet, fail to recognize that a wheel would solve the problem. Reinvention is costly in both time and money. Failure to recognize known design problems can also be costly in usability.

At present, information to support design generation typically takes the form of either heuristic guidelines or general rules of cognition. Heuristic guidelines s pecify particulars of certain "good" designs, but are sporadic and do not allow reasoning about their implications for a specific design situation. For example, "Guideline 1: It is essential that the [Virtual Environment] contain several 1 andmarks" [10]. General rules about human cognition and perception, in contrast, are systematic and allow general reasoning about their relevance, but are often difficult to link to design particulars. For example, "The choice points of a route, at which $\mathrm{n}$ avigation decisions are made, provide a natural

Copyright is held by the author/owner(s).

CHI 2002, April 20-25, 2002, Minneapolis, Minnesota, USA.

ACM 1-58113-454-1/02/0004. segmentation of the route a $\mathrm{s}$ represented in [long term memory]" [3].

Generative design theory is aimed at giving the designer information that has the direct pertinence to design of heuristic guidelines, but the systematic and more general nature of cognitive rules. Design theory is prescriptive rather than explanatory [7], that is, where explanatory theory provides causal accounts of existing phenomena ("Y is thus because of $X^{\prime \prime}$ ), prescriptive theory provides rationales for potential phenomena ("Because of $\mathrm{Y}, \mathrm{Z}$ should be thus"). Generative design theory differs from evaluative design theory in that it seeks to predict what design elements and characteristics will produce a desired outcome, rather predicting the outcome that a given design will produce. In human-computer interaction, evaluative theories, such as GOMS-related theories [4], predict human behavior and performance from design, rather than prescribing design from human performance and behavior as is needed from a generative theory.

The framework presented here represents the beginnings of a generative design theory of navigation. Drawn from information in the extensive literature on spatial cognition and cognitive studies of navigation, the framework focuses on the relationships between cognitive attributes of navigational performance and specific elements of environmental design. At present, only design elements that pertain to locomotion—actual movement—are examined.

\section{A FRAMEWORK FOR LOCOMOTIONAL DESIGN}

The term "navigation" is variously used to denote different activities related to the task of determining where things are and getting to them. Here, it is used to denote the overall

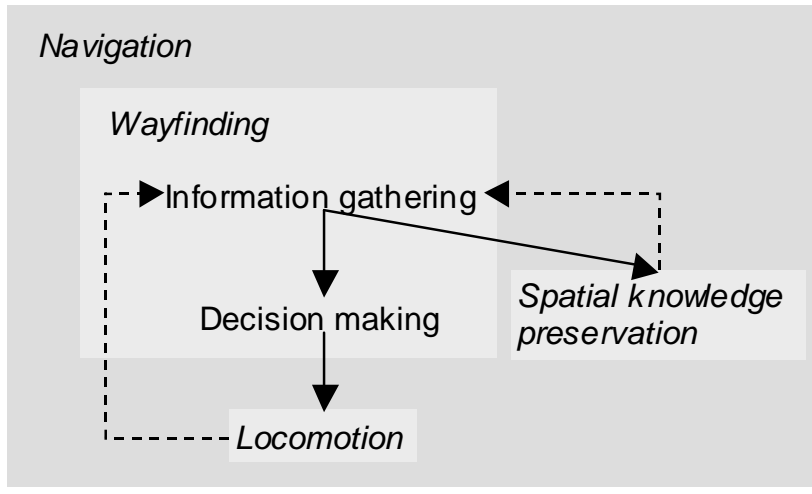

Figure 1 Present t erminology. Solid a rrows indicate d ependencies of activities. Dotted arrows indicate feedback. 
task, which is then decomposed into three subtasks (Figure 1). Wayfinding is the task of spatial problem-solving and decision-making, and is the central-most of the three subtasks. Spatial knowledge preservation is the task of transforming and storing spatial information for future use, including relating $\mathrm{n}$ ew and $\mathrm{p}$ reviously stored information. (Cognitive maps [5] are one well-studied form of such stored information.) Locomotion is the task of actually moving and controlling movement and is the most basic of the three navigational subtasks.

Most work related to locomotion in electronic spaces focuses on the mechanics of controlling movement, e.g., the characteristics and use of different input devices [1] or the input necessary to control complex virtual movement $[6,9]$. The present work sets locomotion in the context of navigation and thus focuses on how environmental design determines what movement is necessary and, consequently, how complex navigation is.

In a navigational context, locomotional design determines what wayfinding decisions must be made, when they must be made, how much time is available to make them and how complex decisions are [3, 8]. (Wayfinding design, in contrast, determines what information is available for making decisions.) Thus a generative theory of locomotional design must prescribe locomotional design elements from wayfinding decision-making processes and performance.

\section{Examples of Elements of Locomotional Design}

Locomotional structure, the set of locations that can be reached and routes between them, is highly significant in determining wayfinding behavior and performance $[5,8]$. In the physical world, design of the locomotional structure is highly limited by the laws of natural physics. However, electronic worlds impose no such restrictions and locations and routes can be designed specifically to meet the needs of the task the design is intended to support and the wayfinding task it incurs. They can even be reorganized, added or eliminated dynamically at low cost.

Thus, the first step in locomotional design is to determine what constitutes a location, what locations are necessary to the task and what connections between them are needed to complete the task. If the design goal is to minimize wayfinding overhead, the designer's objective is to keep the locomotional structure true to this task-defined structure.

Decision points, locations at which decisions are made, are another significant determinant of wayfinding behavior and performance [2, 8]. These can be divided into two types. Branch points are locations where routes intersect [8]. Locomotional decisions must be made before proceeding from a branch point. The designer must balance the number of branch points against the number of options at each branch point in the locomotional structure. Information points are locations at which new information becomes available [2]. The locomotional structure may create information points by revealing views of the environment, as in the physical world when a path crests a hill. Wayfinding design may create a dditional information points by the placement of environmental information such as signage or landmarks. The distance between information and branch point and the speed of movement control the time a vailable to make a decision without interrupting movement.

\section{SUMMARY}

Generative design theories are needed to b ridge the gaps between pure scientific knowledge, individual ("point") designs and systematic generation of viable design alternatives. This paper has laid out the shape of a framework for locomotional design that places movement in a context of navigation and thus uses knowledge of navigation and spatial cognition to inform design. The implications for design of two such pieces of knowledge were sketched out, suggesting how such a framework might lead to design theory.

\section{REFERENCES}

1. Baber, C. (1997). Beyond the Desktop: Designing and Using Interaction Devices. Academic Press.

2. Chown, E., Kaplan, S., Kortenkamp, D. (1995). Prototypes, Location and Associative Networks (PLAN): Towards a Unified Theory of Cognitive Mapping. Cognitive Science 19, 1-51.

3. Golledge, R. G., Smith, T. R., Pellegrino, J. W., Marshall, S. P., Doherty, S. (1985). A Conceptual Model and Empirical Analysis of Children's Acquisition of Spatial Knowledge. Journal of Environmental Psychology, 5. 125-152.

4. John, B. E., Kieras, D. E. (1996). The GOMS Family of User I nterface Analysis Techniques: Comparison and Contrast. ACM Transactions on Computer-Human Interaction, v.3 n.4. 320-351..

5. Lynch, K (1960). The Image of the City. MIT Press.

6. Mackinlay, J. D., Card, S. K., Robertson, G. G. (1990). Rapid Controlled Movement Through a Virtual 3D Workspace. SIGGRAPH '90 Conference Proceedings, in Computer Graphics 24 (4, Aug.), 171-176.

7. Moore, G. T. (1997). Toward Environment-Behavior Theories of the Middle Range. Advances in Environment, Behavior, and Design. Vol 4.

8. Passini, R. (1996). Wayfinding Design: Logic, Application and Some Thoughts on Universality. Design Studies, Vol 17. 319-331.

9. Song, D., Norman, M. (1993). Nonlinear I nteractive Motion Control Techniques for Virtual Space Navigation. 1993 IEEE A nnual Virtual Reality International Symposium. 111-117.

10. Vinson, N. G. (1999). Design Guidelines for Landmarks to Support Navigation in Virtual Environments. CHI 99 Conference Proceedings. 278285. 